\title{
Induction thermography on CFRP and the role of anisotropy
}

by U. Netzelmann* and J. Guo**

*Fraunhofer Institute for Nondestructive Testing IZFP, Dept. Components and Assemblies, Campus E3 1, 66123 Saarbrücken, Germany, udo.netzelmann@izfp.fraunhofer.de

${ }^{*}$ Nondestructive Testing Research Center, School of Physical Science and Technology, Southwest Jiaotong University, Chengdu, PR China

\section{Abstract}

Experiments were performed by induction thermography on carbon fibre reinforced plates. Uni-axial, bi-axial materials as well as woven fabric were studied. The uni-axial material was investigated as a function of the fibre orientation angle and showed heating far away from the inductor and heating patterns with local minima. The occurrence of minima was explained by induction current cancellation effects. The experimentally measured thermal contrast patterns were confirmed by the results of numerical simulations. Fiber breakage could be detected better by induction thermography than by optically excited thermography.

\section{Introduction}

In compound materials with electrically conducting fibres, induction or eddy current thermography can be used for active heating. In carbon fibre reinforced polymer (CFRP) several mechanism of heating are known: resistive heating of the fibres, resistive heating of the contact points between fibres and dielectric heating of crossing points between fibres [1]. Experimentally, induction thermography of CFRP has been performed in early studies [2,3]. Other work on CFRP is cited in [4]. In [4] it was shown that even uni-axially oriented material can be heated by induction. In this work, modeling of CFRP plates was refined and some phenomena were more clearly explained.

\section{Experimental}

An induction system consisting of a frequency generator, a $200 \mathrm{~W}$ broad-band power amplifier, a resonant work-head with various induction coils and a cooled infrared camera was used for the experiments (figure 1). Due to the limited maximum output power of the amplifier, lock-in excitation and processing were used instead of pulsed excitation. The induction frequencies were typically in the range of $170-350 \mathrm{kHz}$.

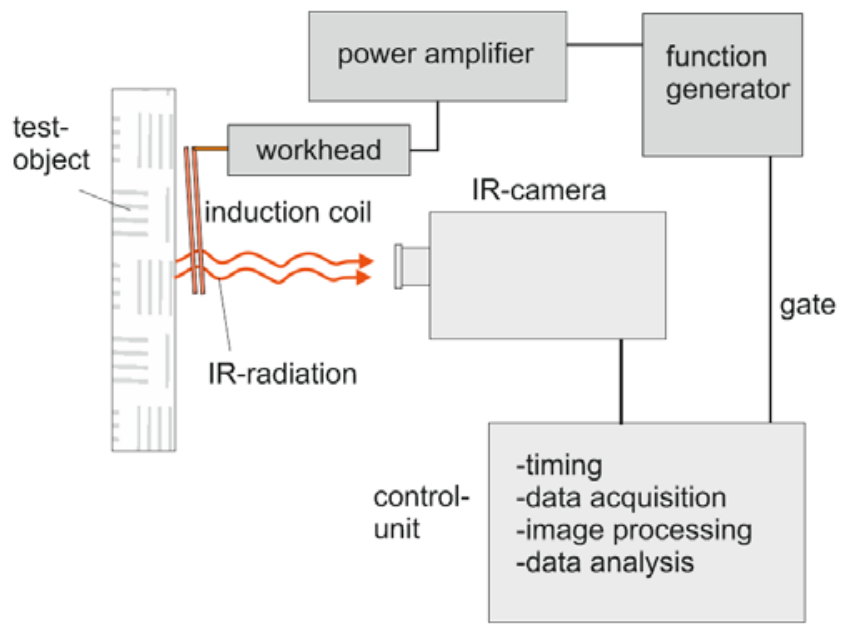

Fig. 1. Experimental setup for the investigations using induction thermography

A QWIP 384 dual-band infrared camera (Thermosensorik) operated in the mid-wave infrared at 4.4 to $5.2 \mu \mathrm{m}$ was employed for the present measurements [5]. Image sequences of either 1024 or 2048 frames were recorded at a frame rate of $145 \mathrm{frames} / \mathrm{s}$. Image recording was synchronized with the rectangular modulated burst excitation. The modulation frequencies $\mathrm{f}_{\mathrm{M}}$ were between 0.4 and $36 \mathrm{~Hz}$. Amplitude and phase images were calculated from the recorded image sequences. 
Due to the low electical conductivity of CFRP the samples used in this study could be assumed to electromagnetically thin. This means that the electromagnetic skin depth is large compared to the geometrical thickness. Using material parameters from literature, a skin depth of $5 \mathrm{~cm}$ can be estimated in the direction perpendicular to the fiber plane at an induction frequency of about $100 \mathrm{kHz}$. This allows one to perform measurements in reflection (the inductor is between sample and IR camera) as well as in transmission arrangement (the inductor is located on the sample side away from the camera).

\section{Results and discussion}

\subsection{CFRP plates with uni-axial anisotropy}

Investigation were performed on a disk of uni-axially oriented CFRP with a thickness of $1 \mathrm{~mm}$ and a diameter of $200 \mathrm{~mm}$. Parallel oriented fiber bundles with a width of about $5 \mathrm{~mm}$ each were embedded in a polyester matrix. A rectangular inductor coil with dimensions of $120 \mathrm{~mm} \times 35 \mathrm{~mm}$ was positioned in the center of the disc. A marker on the disc allowed to set a rotation angle $\beta$ between the long coil axis and the fiber direction of the sample (figure 2).

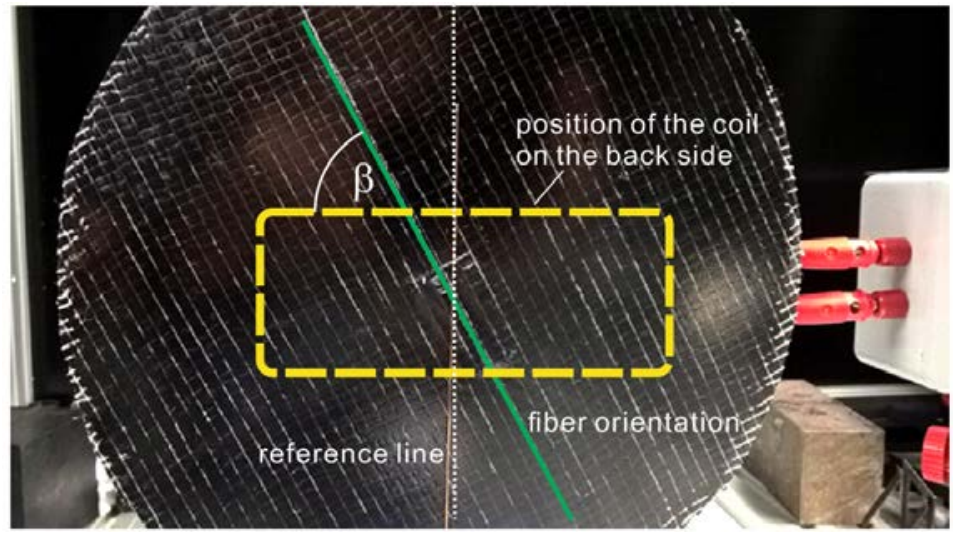

Fig. 2. Photo of the experimental set-up for induction thermography in transmission arrangement. The marker indicates the fiber direction $\beta$

For the following measurements an induction frequency of $329 \mathrm{kHz}$ and a modulation frequency of $\mathrm{f}_{\mathrm{M}}=0.57 \mathrm{~Hz}$ were used. The inductor was positioned in reflection arrangement. Figure 3 shows thermographic amplitude images at different rotation angles $\beta$. Bright grey values correspond to high temperature amplitudes, whereas dark grey values represent low temperature amplitudes. 


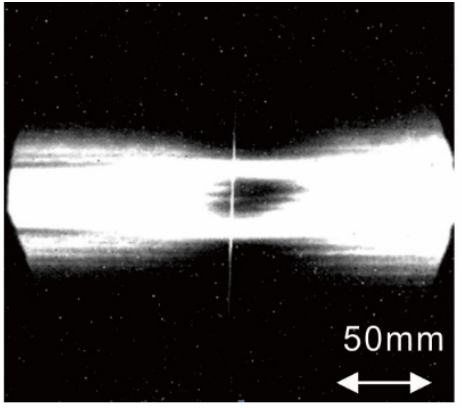

$0^{\circ}$

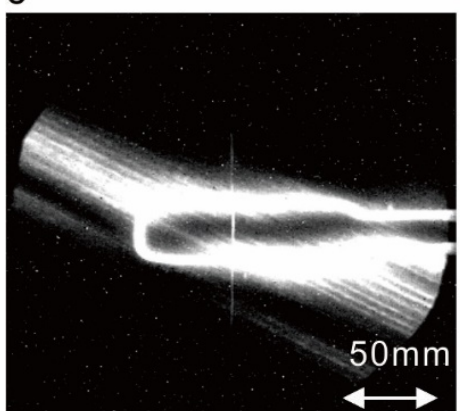

$30^{\circ}$

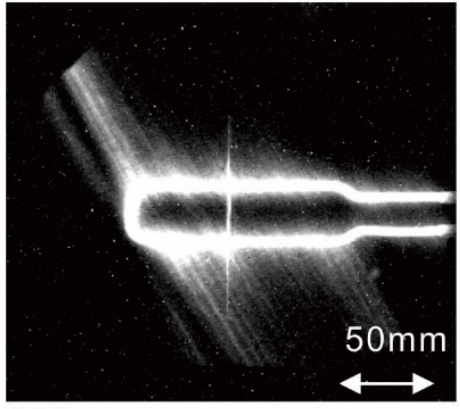

$60^{\circ}$

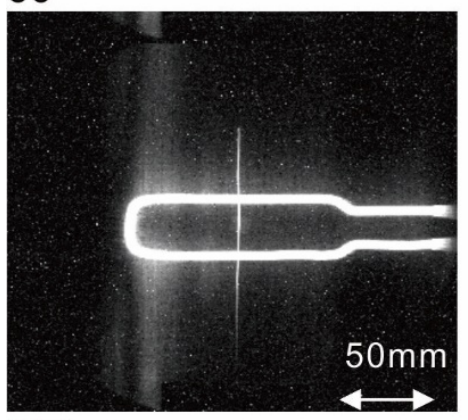

$90^{\circ}$

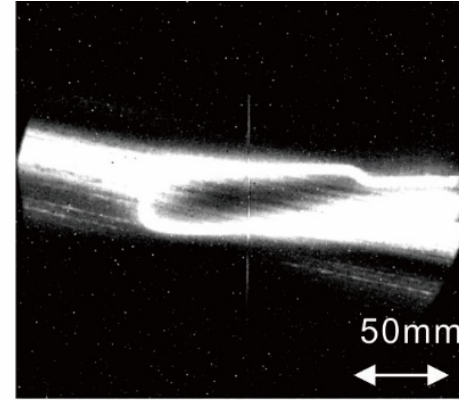

$10^{\circ}$

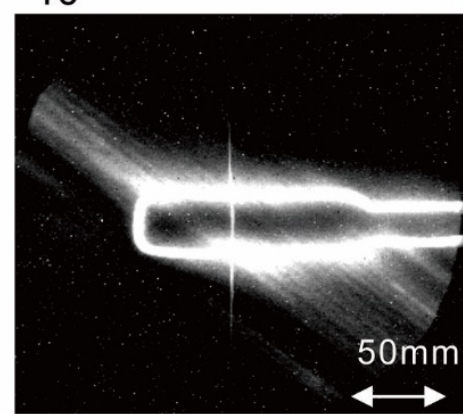

$40^{\circ}$

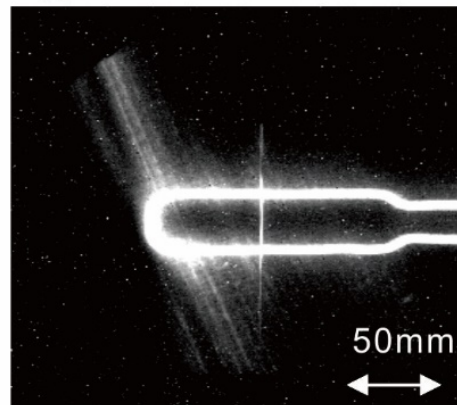

$70^{\circ}$

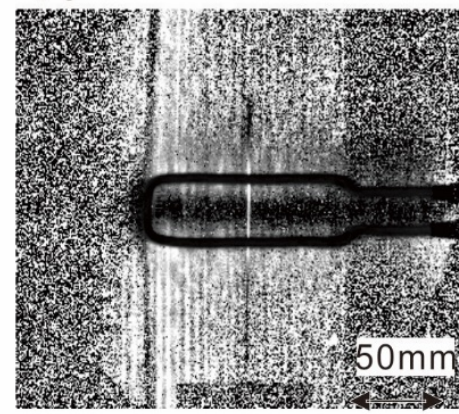

$90^{\circ}$, phase image

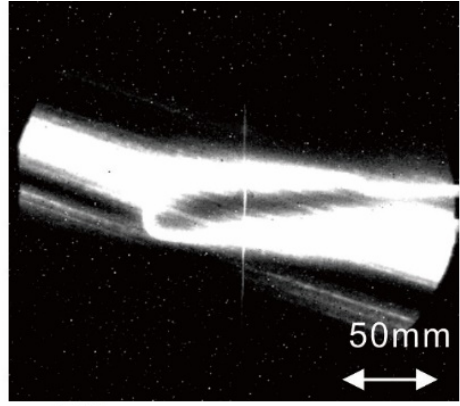

$20^{\circ}$

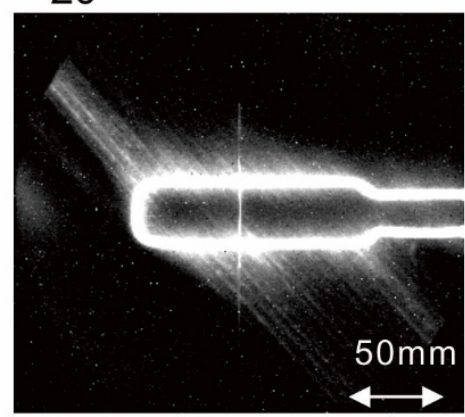

$50^{\circ}$

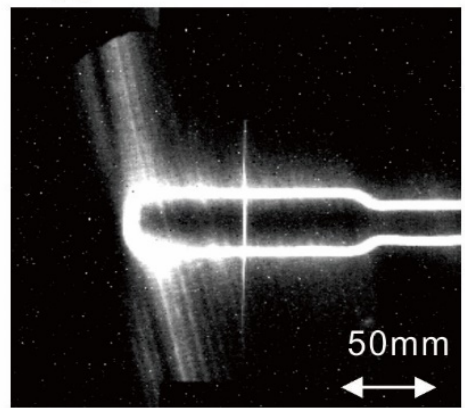

$80^{\circ}$

Fig. 3. Thermographic amplitude images of the uni-axial sample for various rotation angles $\beta$. For $\beta=90^{\circ}$, a phase image is shown in addition. The thin vertical line is a copper wire is a reference that is perpendicular to the long coil axis

The largest temperature oscillations were observed at the angle of $\beta=0^{\circ}$, when the long side of the coil was parallel to the fiber direction. But also for non-zero angles there was still significant heating of the fibres. Current flow usually extended from the coil to the outer rim of the sample disc. Close to the coil, this heating pattern was observable even at $\beta=90^{\circ}$. The corresponding phase image shows that current is flowing far from the coil. Similar results were observed in previous studies on eddy current testing of uni-axial CFRP [6]. 

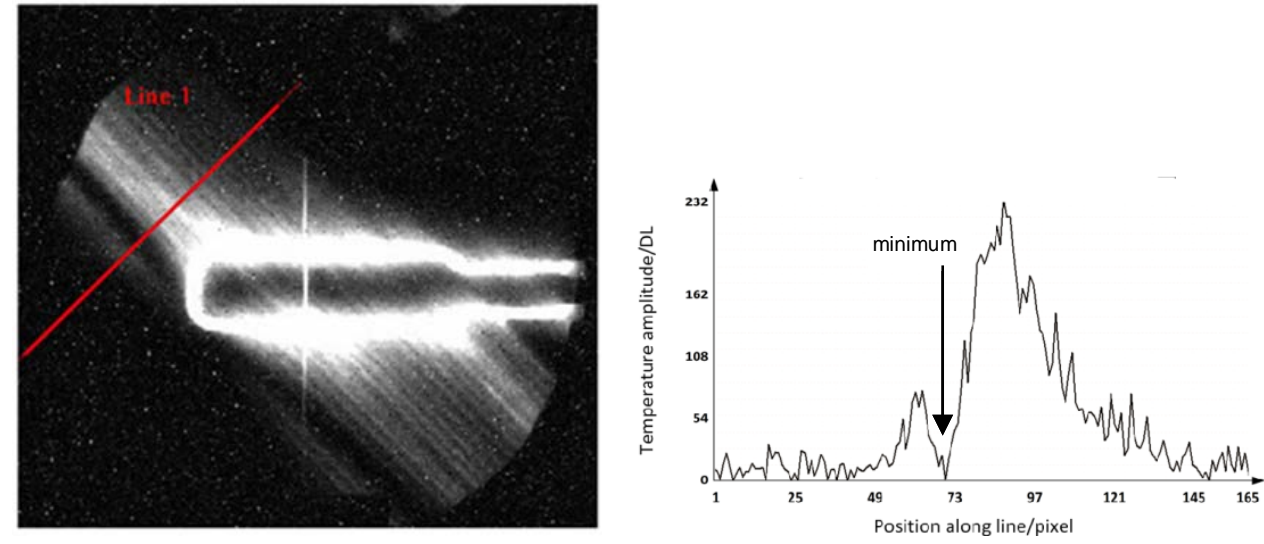

Fig. 4. Left: Thermographic amplitude image at $\beta=45^{\circ}$. Right: Signal line profile perpendicular to the fiber direction along the red line. A minimum is observed at pixel position 70

Close to the short edge of the coil a minimum of heating was observed along a line in fiber direction (figure 4) This effect occurs at all values of $\beta$ and will be explained later. As the thermal signal phase does not show a significant effect close to the minimum line, one can assume an electromagnetic origin of this effect.

In order to gain deeper insight to the current flow in the anisotropic material, finite element simulations using COMSOL Multiphysics 5.2 were performed for a circular coil over an uni-axial anisotropic CFRP sample with dimensions of $100 \mathrm{~mm} \times 100 \mathrm{~mm} \times 1 \mathrm{~mm}$. For simplification, the sample was modeled as a homogeneous anisotropic medium. The effective material parameters were taken from literature [7] and are listed in table 1. In this case, dielectric losses were not assumed, as the frequencies were rather low $(100 \mathrm{kHz})$.

Table 1. Material parameters used for simulation of the uni-axially anisotropic CFRP plate

\begin{tabular}{|c|c|c|c|c|c|c|}
\hline parameter & $\begin{array}{l}\text { density } \\
\text { in } \mathrm{kg} / \mathrm{m}^{3}\end{array}$ & $\begin{array}{l}\text { specific } \\
\text { heat } \\
\text { capacity in } \\
\mathrm{J} /(\mathrm{kg} \cdot \mathrm{K})\end{array}$ & $\begin{array}{l}\text { thermal } \\
\text { conductivity in } \\
\mathrm{W} /(\mathrm{m} \cdot \mathrm{K})\end{array}$ & $\begin{array}{l}\text { electrical } \\
\text { conductivity in } \\
\text { S/m }\end{array}$ & $\begin{array}{l}\text { relative } \\
\text { permeability }\end{array}$ & $\begin{array}{l}\text { relative } \\
\text { dielectric } \\
\text { constant }\end{array}$ \\
\hline x-direction & \multirow{3}{*}{1500} & \multirow{3}{*}{1250} & 6 & 20000 & \multirow{3}{*}{1} & \multirow{3}{*}{1} \\
\hline$y$-direction & & & 0.7 & 100 & & \\
\hline z-direction & & & 0.7 & 100 & & \\
\hline
\end{tabular}

A first model calculation tries to show some basic properties of the current flow in the uni-axial material. In figure 5 , the calculated current density at the surface of the plate is shown. The fiber direction was the $x$-direction. The position of the circular coil is indicated. 

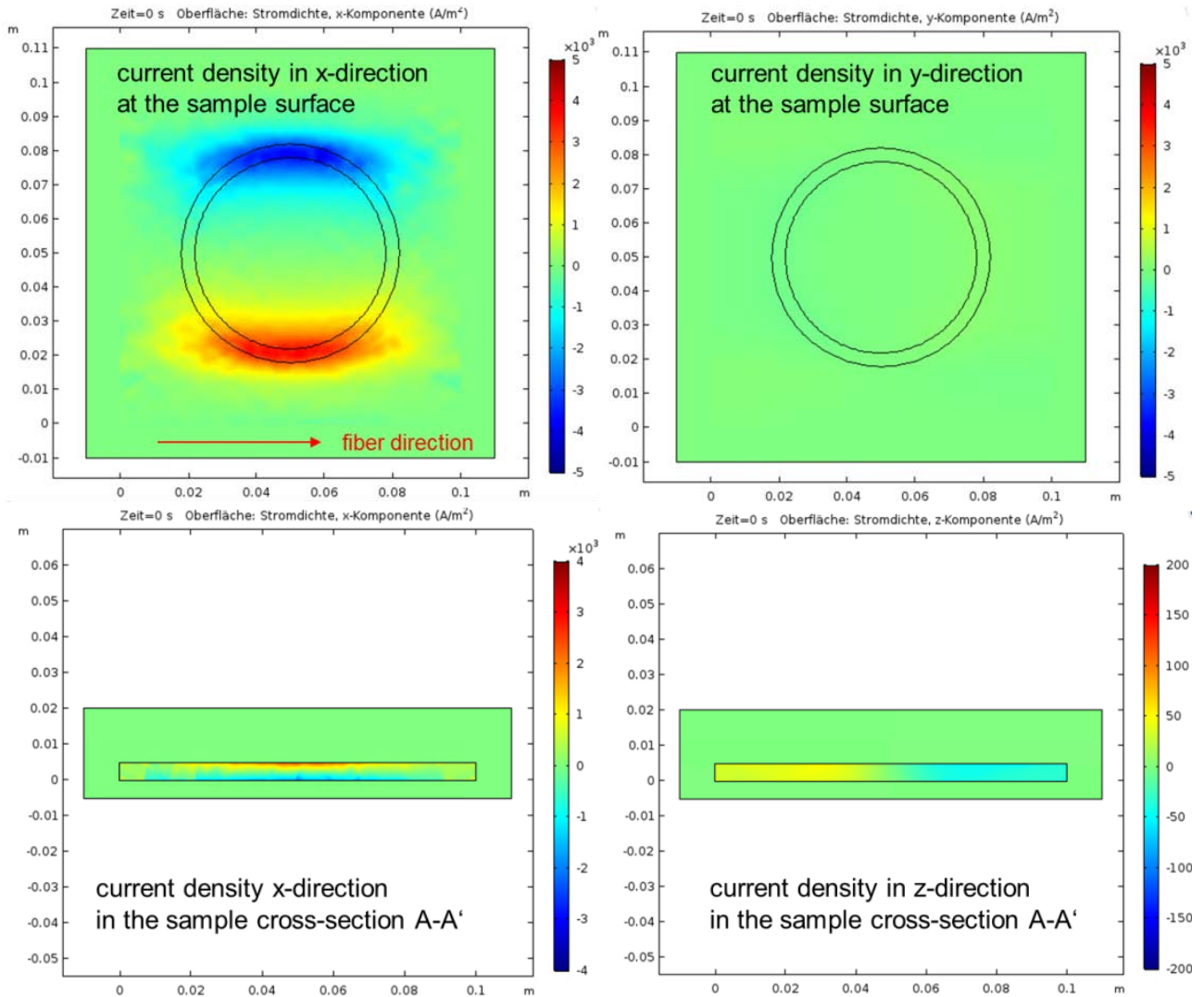

Fig. 5. Simulated induced current density in $x$ - and $y$-directions at the sample surface (top row) and in $x$ - and zdirection in the sample cross-section. The red marker indicates the main current line in the cross-section

Looking first at the induced current at the sample surface (top row of figure 5) it can be seen that the current in $x$-direction follows the fiber direction. There is nearly no current in the $y$-direction. On the other hand, the current lines must be closed. The current profiles in the cross-section of the sample at a position $A A^{\prime}$ (bottom row of figure 5) indicate that over the sample thickness there is a positive current in the x-direction on the induction coil side, but a negative current on the opposite side. There is also a z-component of the current in the cross-section which is positive in the left half and negative in the right half of the sample. In total, the current lines are not closed in the sample surface, but in the cross-section of the sample.

The experiment shown in figures 3 and 4 was then modeled in more detail. The sample disc and the rectangular coil were considered with their true dimensions (figure 6)

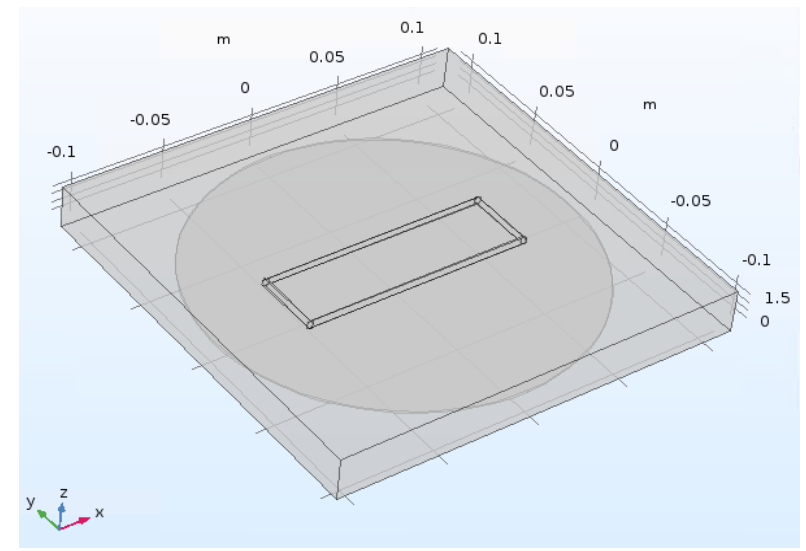

Fig. 6. Sketch of the sample modelled 


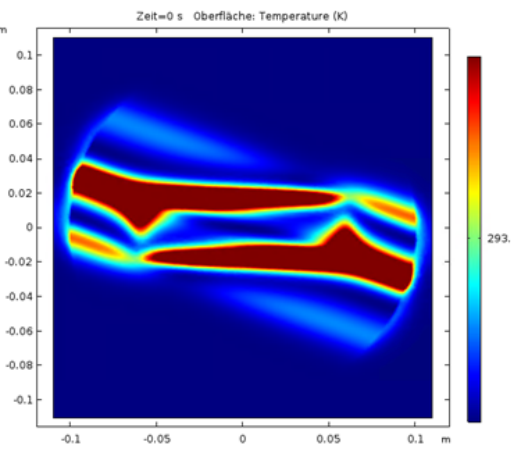

$\beta=20^{\circ}$

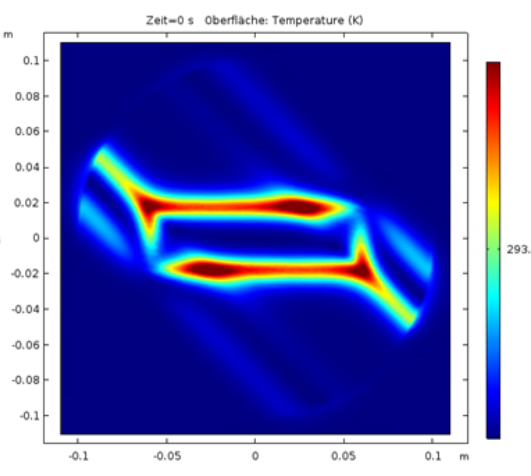

$\beta=45^{\circ}$

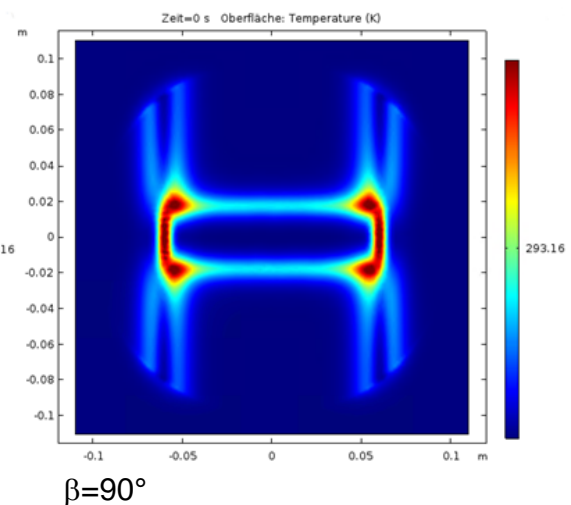

$\beta=90^{\circ}$

Fig. 7. Simulated surface temperature distribution by a rectangular coil at different rotation angles $\beta$ to be compared with experimental data shown in figures 3 and 4

Figure 7 shows the modelling results of the temperature distribution for three different rotation angles $\beta$ of the fiber direction. They have to be compared with the corresponding experimental results from figures 3 and 4 . There is a good agreement between experiment and simulation. In particular, the temperature minima are reproduced well.

The following graph (figure 8) tries to explain the generation of signal minima. At the corner of an inductor coil the coil current in two coil elements A and B has the same absolute value, but changes the direction. Each coil current can be separated into a component in fiber direction (horizontal) and perpendicular to the fiber direction (vertical). One can now consider the induced current from the coil elements $A$ and $B$, called $A^{\prime}$ and $B^{\prime}$. The absolute of the induced current is the smaller, the larger the distance from the coil element is. More current is induced in the fiber direction than in the weakly conducting direction perpendicular to the fibers. The vector sum of the induced currents $A^{\prime}$ and $B^{\prime}$ will largely cancel at positions where the distance to the coil elements is the same. A small current contribution in the direction perpendicular to the fiber will remain. But as the thermal heat source is proportional to the square of the induction current, this contribution will be small in the thermal image and appear as a clear minimum.

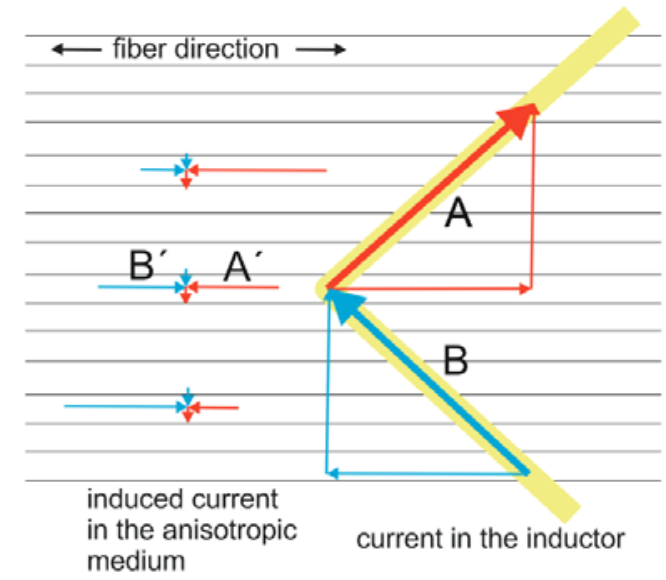

Fig. 8. Sketch for explanation of the formation of signal minima

The signal minima are a consequence of the material anisotropy and cancellation of currents. In an isotropic material the cancellation is not observed, as there will always be current flow components in different directions, which are not cancelled completely.

\subsection{CFRP sample with ply stacks $0^{\circ} 190^{\circ}$}

A second sample investigated consisted of six plies, where two of them were oriented in $0^{\circ}$ direction, two in $90^{\circ}$ direction and two again in $0^{\circ}$ direction (figure 9). The dimensions were $100 \mathrm{~mm} \times 100 \mathrm{~mm}$, the total thickness was $1.6 \mathrm{~mm}$. 


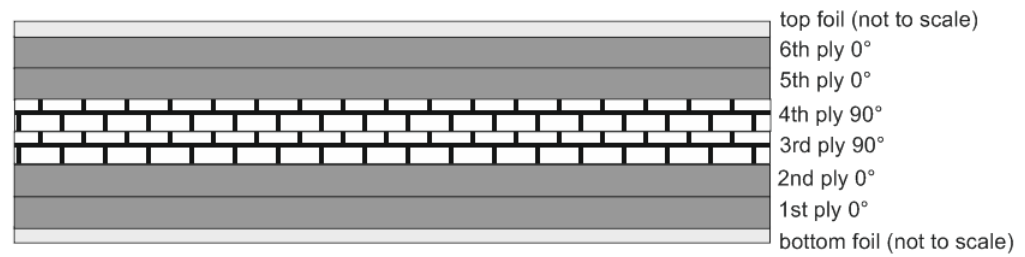

Fig. 9. Layer structure of the sample investigated

In the experiment, the sample was investigated both in transmission and reflection mode. Again, a pronounced heating pattern can be seen. In figure 10, thermal amplitude images in transmission and reflection measured at a modulation frequency of $\mathrm{f}_{\mathrm{M}}=0.57 \mathrm{~Hz}$. The induction coil was the same as described before.
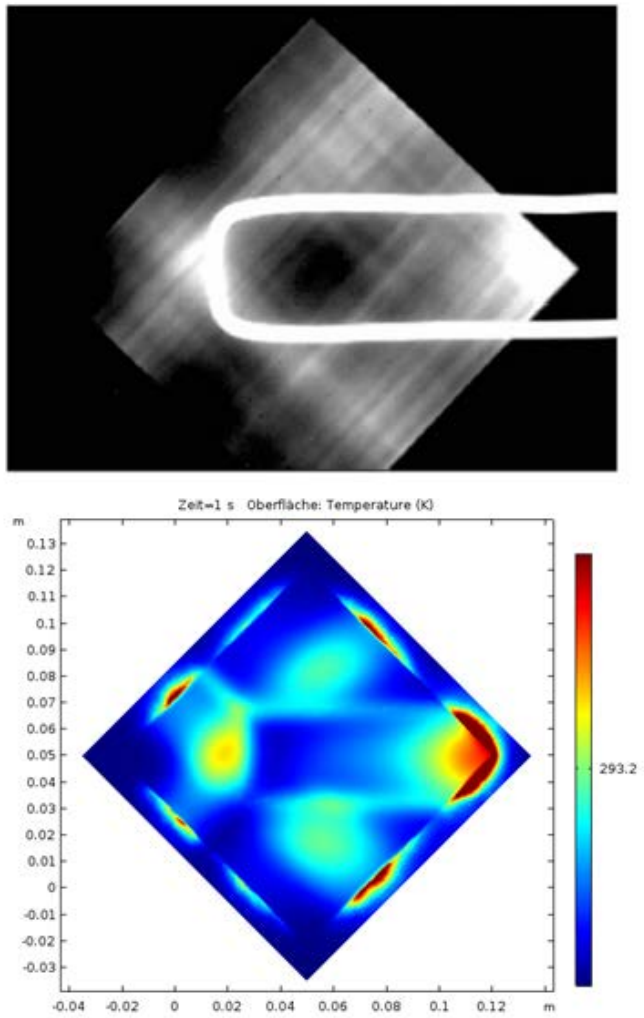
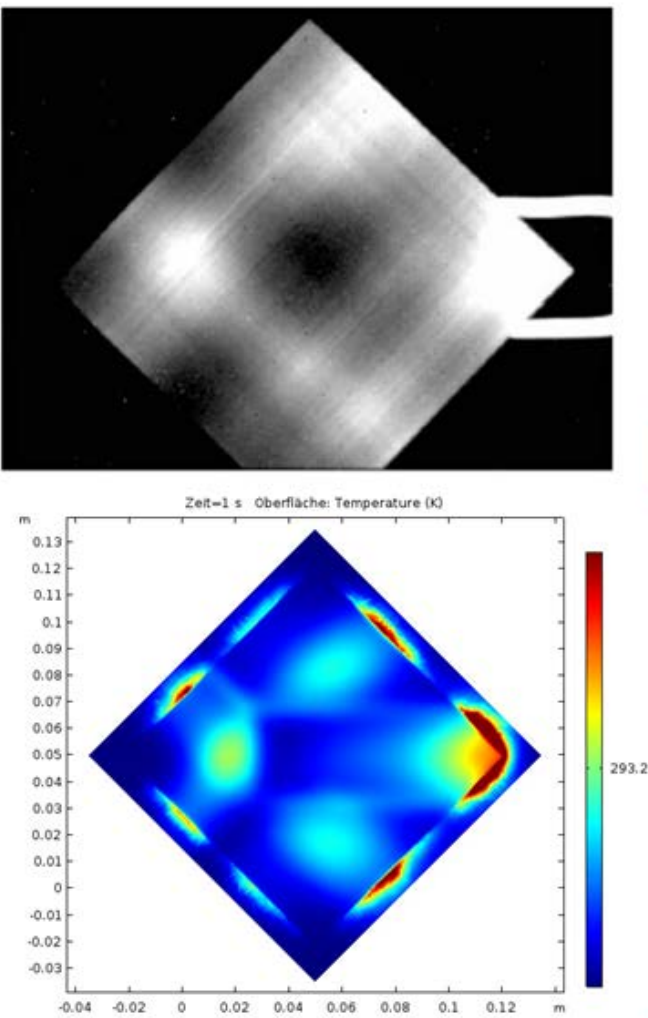

Fig. 10. Top row: Thermographic amplitude images in reflection (left) and transmission (right) arrangement. Bottom row: results of the simulated heating patterns in reflection (left) and transmission (right) in the $0^{\circ} \% 0^{\circ}$ oriented sample after $1 \mathrm{~s}$ of heating. The main fiber orientation of the sample was rotated $45^{\circ}$ relative to the long axis of the coil

For a corresponding numerical simulation, a thin layer on dielectric matrix material between the carbon fibre layers had to be assumed in order to obtain a reasonable agreement of the calculated heating patterns with the experiment. The dielectric layer allowed capacitive coupling between the plies, but it was not necessary to assume dielectric losses in this layer. The heating patterns correspond well with the experimental results. Heating is observed also at the outer rim of the sample. The samples appear to be transparent for the electromagnetic excitation, which follows from the similar contrast obtained in reflection and transmission arrangement.

\subsection{Multi-ply woven fabric with impact damage}

Further samples investigated consist of 19 plies of $0^{\circ} / 90^{\circ}$-fiber rovings, which were embedded in a polypropylene-disulfide matrix with a fiber volume content of $52 \%$. The sample dimensions were $150 \mathrm{~mm} \times 100 \mathrm{~mm} \times$ $4 \mathrm{~mm}$. The samples had been damaged by impacts at various energies fom 1 to $10 \mathrm{~J}$. In this case, the induction frequency was $329 \mathrm{~Hz}$, the modulation frequency was $\mathrm{f}_{\mathrm{M}}=1.13 \mathrm{~Hz}$. Beside inductive heating, for comparison another set 
of experiments was performed with periodic broad optical illumination by two LED arrays at 940 nm wavelength operated the same modulation frequency of $1.13 \mathrm{~Hz}$. In figure 11, a thermal phase image with inductive heating and thermal amplitude and phase images with LED optical heating are shown from the same sample region around a impact damaged zone. The results obtained by induction heating suggest that at least partly broken fibers are present. The contrast in the phase image resembles typical contrasts obrained from surface cracks in metals. Reference measurements by x-ray CT proved the existence of fiber cracking and showed inner delaminations as known to be generated by impacts [4]. The damaged region is very clearly visible in the induction thermography phase image, usually better than in the corresponding amplitude image. Cracking is less visible in the optically excited amplitude image and only very weakly in the corresponding phase image. In the latter, the contrast is superimposed by the strong contrast from the weaving structure.

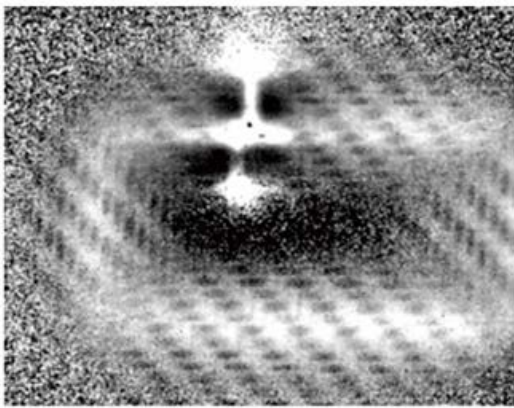

a)

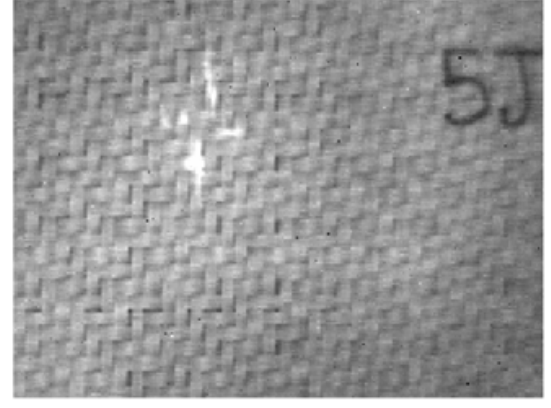

b)

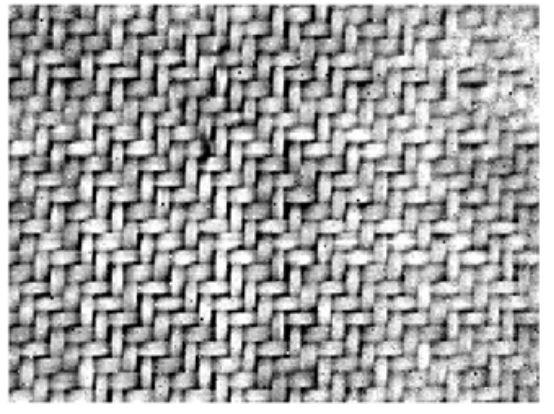

c)

Fig. 11. a) Thermographic phase image by induction thermography from an impacted CFRP sample. b) the corresponding thermal amplitude image obtained by heating using an LED array. c) the corresponding thermal phase image obtained by heating with a LED array

\section{Conclusion}

When testing uni-axial CFRP, one can expect that induction currents will flow even far away from the inductor, until they meet the edge of the test object. This occurs at all angles between the fiber direction and the long axis of a rectangular coil. Close to the edges of the rectangular coil mimima of heating were found. They can be explained by cancellation effects due to different current contributions induced in opposite direction. Finite-element simulations did reveal similar effects. Such minima may cause dead zones in thermographic NDT.

In bi-axially oriented samples heating far from the inductor coil could be observed as well, but not the minima along lines. Measurements on woven multi-ply impact damaged CFRP by induction thermography in comparison to LED array excited thermography showed, that induction thermography allows sensitive detection of fiber breakage. This is a contrast complementary to well-known contrast due to internal delaminations, which is accessible by optically excited thermography.

\section{REFERENCES}

[1] Yarlagadda S, Kim HJ, Gillespie JW, Shevchenko NB, Fink BK. A Study on the Induction Heating of Conductive Fiber Reinforced Composites. Journal of Composite Materials. 2002;36:401-21

[2] Lehtiniemi R, Hartikainen J. An application of induction heating for fast thermal nondestructive evaluation. Review of Scientific Instruments. 1994;65:2099-101

[3] Shepard S M, Ihota J R, Ahmed T. Development of NDE Technique with Induction Heating on Conductive Composite Material. USAF Report Number AFRL-SR-TR-05-0043 (2004)

[4] Guo J, Gao X, Toma E, and Netzelmann U. Anisotropy in carbon fiber reinforced polymer (CFRP) and its effect on induction thermography. NDT \&E International. 2017; 91:1-8

[5] Abuhamad $M$ and Netzelmann U, 'Dual-band active thermography on infrared transparent materials', Proc. 10th Int. Conf. on Quantitative Infrared Thermography (QIRT), 26.-30.7.2010, Quebec City, Canada, p. 868-875

[6] Mook G, Lange R, Koeser O, 'Non-destructive characterization of carbon fibre reinforced plastics by means of eddy-currents', Composites Science and Technology 61 (2001) 865-873

[7] Karch C, Wulbrand W, Stössel R, 'Induktions- und Blitzlichtthermographie -Modellierung und Simulation-', Thermographie Kolloquium 2011, 29.-30.9.2011, Stuttgart, DGZfP Berichtsband BB 130-CD, V21 (2011) 